\title{
Serum Decorin and Biglycan as Potential Biomarkers to Predict PPROM in Early Gestation
}

\author{
Lori A. Underhill, PhD ${ }^{1,2}$, Nora Avalos, BS $^{2}$, Richard Tucker, BS ${ }^{2}$, \\ Zheng Zhang, $\mathbf{P h D}^{3}$, Geralyn Messerlian, $\mathbf{P h D}^{2}$, and \\ Beatrice Lechner, MD ${ }^{1,2}$
}

\begin{abstract}
Preterm birth is a leading cause of neonatal mortality in the US and globally, with preterm premature rupture of fetal membranes (PPROM) accounting for one third of preterm births. Currently no predictive diagnostics are available to precisely assess risk and potentially reduce the incidence of PPROM. Bigycan and decorin, the main proteoglycans present in human fetal membranes, are involved in the physiological maturation of fetal membranes as well as in the pathophysiology of preterm birth. The serum protein sex hormone-binding globulin (SHBG) has recently been identified as a predictor of spontaneous preterm birth. We hypothesize that the balance between serum decorin and biglycan on one hand and SHBG on the other hand may provide insight into the status of the fetal membranes in early pregnancy, thereby predicting PPROM prior to symptoms. Using chart review, I 8 patients with confirmed cases of PPROM were identified from 2013-2016. Second trimester residual serum was retreived from freezer storage for these cases along with 5 matched controls for each case. The biomarkers biglycan, decorin and SHBG were analyzed first separately, then in combination to determine their ability to predict PPROM. The predictive score for the combined model displays an AUC $=0.774$. The ROC curve of the predicted score has an optimal threshold of 0.238 and a sensitivity and specificity of 0.72 and 0.84 respectively. This prenatal serum panel is a promising serum screening-based biochemical model to predict PPROM in asymptomatic women.
\end{abstract}

\section{Keywords}

biglycan, decorin, PPROM, biomarkers

\section{Introduction}

Preterm birth is a leading cause of neonatal mortality and morbidity in the United States and globally, with preterm premature rupture of fetal membranes (PPROM) accounting for about one-third of preterm births. ${ }^{1-3}$ PPROM is the spontaneous rupture of fetal membranes prior to labor before 37 weeks of gestation. ${ }^{4}$ The etiology of PPROM is multifactorial, with nutritional, environmental, and genetic contributors working singularly or synergistically. ${ }^{5,6}$ Clinical risk factors have been proposed to identify women at highest risk of PPROM, including short cervical length at 23 to 24 weeks gestation, positive fetal fibronectin screening, and a history of prior PPROM. ${ }^{7}$ These clinical risk factors generate PPROM through pathways involved in the upregulation of the inflammatory process. ${ }^{5}$ The resulting inflammation can be microbial-associated or nonmicrobial-associated (sterile), both equally common in PPROM after 25 weeks gestation. ${ }^{8}$ Currently, no predictive diagnostics, such as serum biomarkers, have been identified that would more precisely assess risk and potentially reduce the incidence of PPROM.
Infants born with Ehlers-Danlos syndrome (EDS), a genetic connective tissue disorder, have an increased risk of PPROM compared to their unaffected siblings. ${ }^{9,10}$ One subtype of EDS is caused by a mutation that leads to the abnormal secretion of biglycan and decorin, ${ }^{11}$ two small leucine-rich proteoglycans (SLRPs) that are components of the extracellular matrix (ECM) of most tissues. They are known to play a significant role in the tensile strength of connective tissues. ${ }^{12-14}$ Additionally, biglycan plays a role in the inflammatory cascade as a ligand for the Toll-like-receptor 2 and $4 .{ }^{15}$ Biglycan and decorin are the main proteoglycans present in the intermediate and reticular layers of human fetal membranes. ${ }^{16}$ We have previously shown that

\footnotetext{
' Warren Alpert Medical School at Brown University, Providence, RI ,USA

${ }^{2}$ Department of Pediatrics, Women and Infants Hospital, Providence, RI, USA

${ }^{3}$ School of Public Health, Brown University, Providence, RI, USA
}

\section{Corresponding Author:}

Lori A. Underhill, PhD, Women \& Infants Hospital, I0I Dudley Street, Providence, RI, 02905, USA.

Email: Lunderhill@wihri.org 
biglycan and decorin transcriptionally compensate for each other during fetal membrane maturation but lose their compensatory activity in the presence of inflammation. ${ }^{17}$ Biglycan and decorin have also been shown to be involved in the pathophysiology of preterm birth, with dysregulation of decorin occurring in fetal membranes during the second trimester. ${ }^{18,19}$ We have shown that mice deficient in biglycan and decorin, an animal model of EDS, deliver their pups prematurely and display morphologic as well as signaling abnormalities in their fetal membranes. ${ }^{20,21}$

The serum protein sex hormone-binding globulin (SHBG) has recently been identified as a predictor of spontaneous preterm birth (sPTB). ${ }^{22}$ Plasma SHBG levels increase significantly during pregnancy, ${ }^{23}$ but are downregulated in spontaneous preterm delivery. Spontaneous preterm births include deliveries resulting from spontaneous preterm labor (PTL) and/or PPROM. ${ }^{24}$ Preterm labor is defined as regular contractions of the uterus with or without intact fetal membranes before 37 weeks of pregnancy. PPROM is defined by the rupture of fetal membranes prior to the onset of labor. PPROM does not occur in all cases of SPTB, suggesting that the underlying pathophysiology of PPROM and spontaneous PTL may be distinct. ${ }^{25,26}$

We hypothesize that the balance between decorin and biglycan on one hand, and SHBG on the other hand in the serum may provide insight into the tensile and inflammatory status of the fetal membranes in the clinically asymptomatic participant, thereby functioning as a PPROM biomarker panel prior to symptoms.

The clinical importance of predicting PPROM before it occurs lies in the fact that once PPROM has occurred, it cannot be reversed and will ultimately lead to preterm birth. Thus, a biomarker panel for PPROM would allow for the development of targeted interventions in pregnancies deemed at high risk for PPROM.

\section{Materials and Methods}

\section{Sample Collection}

All serum samples were collected from patients who presented to Women and Infants Hospital, Providence, Rhode Island, for second-trimester screening. This retrospective study was conducted with approval from the Care New England Institutional Review Board.

For the preanalytical and covariate studies, samples were selected from singleton pregnancies in order to assess the effects of gestational age (weeks 15-20), freezer storage $(0$, 6 , or 12 months), and maternal race (African American or Non-African American) on biglycan and decorin serum values. Serum samples from nonpregnant women were chosen by a negative human chorionic gonadotrophin result.

For the biomarker study, using chart review, the first 18 patients with PPROM going backward temporally starting from 2016 were identified. All samples for this study were from 2013 to 2016. PPROM was defined as the presentation of rupture of fetal membranes/leaking of amniotic fluid as the first concerning sign or symptom in an otherwise uncomplicated pregnancy. Cases in which contractions, pain, or infection were documented as a symptom prior to or coinciding with the PPROM or amniotic fluid leakage were excluded as were multiples, congenital anomalies, genetic diagnoses, maternal drug use, the use of artificial reproductive technologies, and PPROM at less than 17 weeks gestational age. The resulting gestational age at diagnosis for the PPROM cases ranged from 17 to 27 weeks.

Among the confirmed cases of PPROM, we searched to find 18 women who had a standard prenatal serum screening test for aneuploidy in that pregnancy. Second-trimester residual serum was retrieved from $-20^{\circ} \mathrm{C}$ freezer storage for these 18 cases, along with 5 matched controls for each case. The control samples were matched for gestational age at sample collection (within the same completed week, 15-20 weeks of gestation), maternal race (African American or non-African American), and duration of freezer storage ( \pm 6 months). None of the 108 patients were smokers.

\section{Assays}

Alpha-fetoprotein (AFP) levels were measured at the time of sample receipt using an automated chemiluminescent assay kit purchased from Beckman Coulter, Chaska, Minnesota. The SHBG levels were tested for this study after a sample thaw using an automated immunoassay method from Siemens Diagnostics, on the Immulite 2000, Los Angeles, California. These analytes are routinely measured in the clinical laboratory at Women and Infants Hospital in accordance with CAP and CLIA guidelines.

Serum biglycan and decorin were measured by commercially available enzyme-linked immunosorbent sandwich assay (ELISA) kits (Human Biglycan ELISA kit; Clone-Cloud Corp, Houston, Texas) and (Human Decorin ELISA kit; SigmaAldrich, St. Louis, Missouri), respectively. The assays were performed according to manufacturer's instructions. Briefly, a monoclonal capture antibody is precoated onto 96-well plates. The biological sample and standard are then added to the well, and incubated. Biotin-labeled detection antibody is then added to the well, followed by incubation with horseradish peroxidase streptavidin. Unbound conjugates are washed away, followed by colorimetric analysis on a BioRad IMark Microplate Reader (BioRad, Hercules, California) at $450 \mathrm{~nm}$. Analytical validation of the biglycan and decorin assays was carried out prior to the serum sample biomarker analysis and included patient interassay variation, linearity, and limit of detection. Testing of serum samples was performed without operator knowledge of PPROM status.

\section{Statistics}

Median concentrations were calculated for biglycan, decorin, AFP, and SHBG for all analyses. In the preanalytical and covariate studies, nonparametric analysis of group differences was calculated by Wilcoxon test. For more than 2 groups, a post hoc Kruskal-Wallis test was performed. 

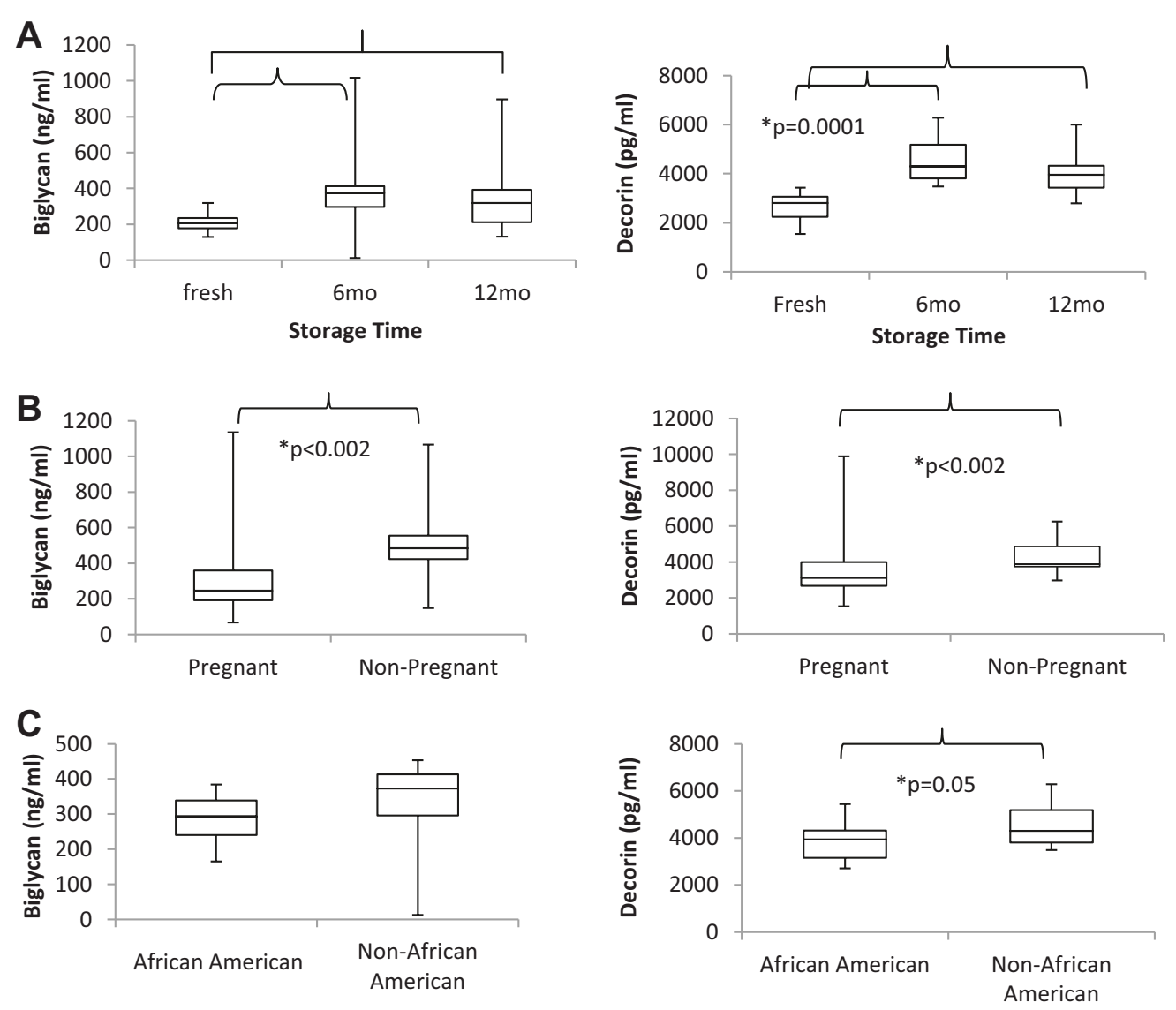

Figure I. Covariate study variables. A, Median serum levels of both biglycan and decorin were lower in pregnant than nonpregnant women $(P<$ .002). B, Levels of decorin were slightly lower in African American than non-African American women $(P=.047)$, while biglycan levels did not differ by ethnicity. $\mathrm{C}$, Biglycan and decorin show significant differences in serum concentrations after $-20^{\circ} \mathrm{C}$ freezer storage.

For the biomarker study, data were matched for gestational age and race in a 1:5 case-control study design. Negative binomial models for counts were generated for each protein and adjusted for clustering in matched groups using generalized estimating equations which take into account the similarity of participants within the matched groups.

The receiver-operating characteristic (ROC) curve was constructed nonparametrically, and the area under the curve (AUC) was calculated based on the trapezoid rule. The confidence interval was based on the Delong, Delong method. ${ }^{27}$ The predicted score was based on the logistic regression model, and AUC of the predicted score was calculated similarly. The optimal threshold and its associated sensitivity and specificity were calculated using the Youden index and the top left criteria. The confidence intervals of the AUCs and the thresholds were derived from the bootstrap resampling method.

\section{Results}

\section{Preanalytical Study}

The interassay variation was calculated as the mean Coefficient of Variation\% between 8 individual runs and determined to be
Table I. Median Values for Biomarkers in PPROM and Controls.

\begin{tabular}{|c|c|c|c|}
\hline & PPROM $(n=18)$ & Controls $(n=90)$ & \\
\hline Variable & Median (range) & Median (range) & Value \\
\hline $\begin{array}{l}\text { Biglycan, } \\
\mathrm{ng} / \mathrm{mL}\end{array}$ & $428.17(101.05-1121.75)$ & $300.45(44.2-1200)$ & .009 \\
\hline $\begin{array}{c}\text { Decorin, } \\
\mathrm{pg} / \mathrm{mL}\end{array}$ & 2999.1 (2325.8-4352.7) & 3365 (1859.4-6203.8) & .009 \\
\hline $\begin{array}{l}\text { SHBG, } \\
\text { nm/L }\end{array}$ & $347(205-680)$ & $437.75(240-880)$ & .006 \\
\hline AFP, MoM & $1.05(0.66-2.91)$ & $0.98(0.47-2.72)$ & .153 \\
\hline
\end{tabular}

Abbreviation: AFP, alpha-fetoprotein; PPROM, preterm premature rupture of fetal membrane; SHBG, sex hormone-binding globulin.

$5.6 \%$ and $6.9 \%$ for decorin and biglycan, respectively. Assay linearity was calculated as the mean linearity percent (observed value $\times$ the dilution factor of the sample/neat sample value) for 3 random samples. Mean linearity was determined to be $102 \%$ for decorin and $105 \%$ for biglycan. Levels of both decorin and biglycan were increased with 6 months $(\mathrm{n}=15)$ and 12 months $(\mathrm{n}=15)$ of freezer storage $(P=.001)$ and $(P=.03)$, respectively (Figure 1C). 

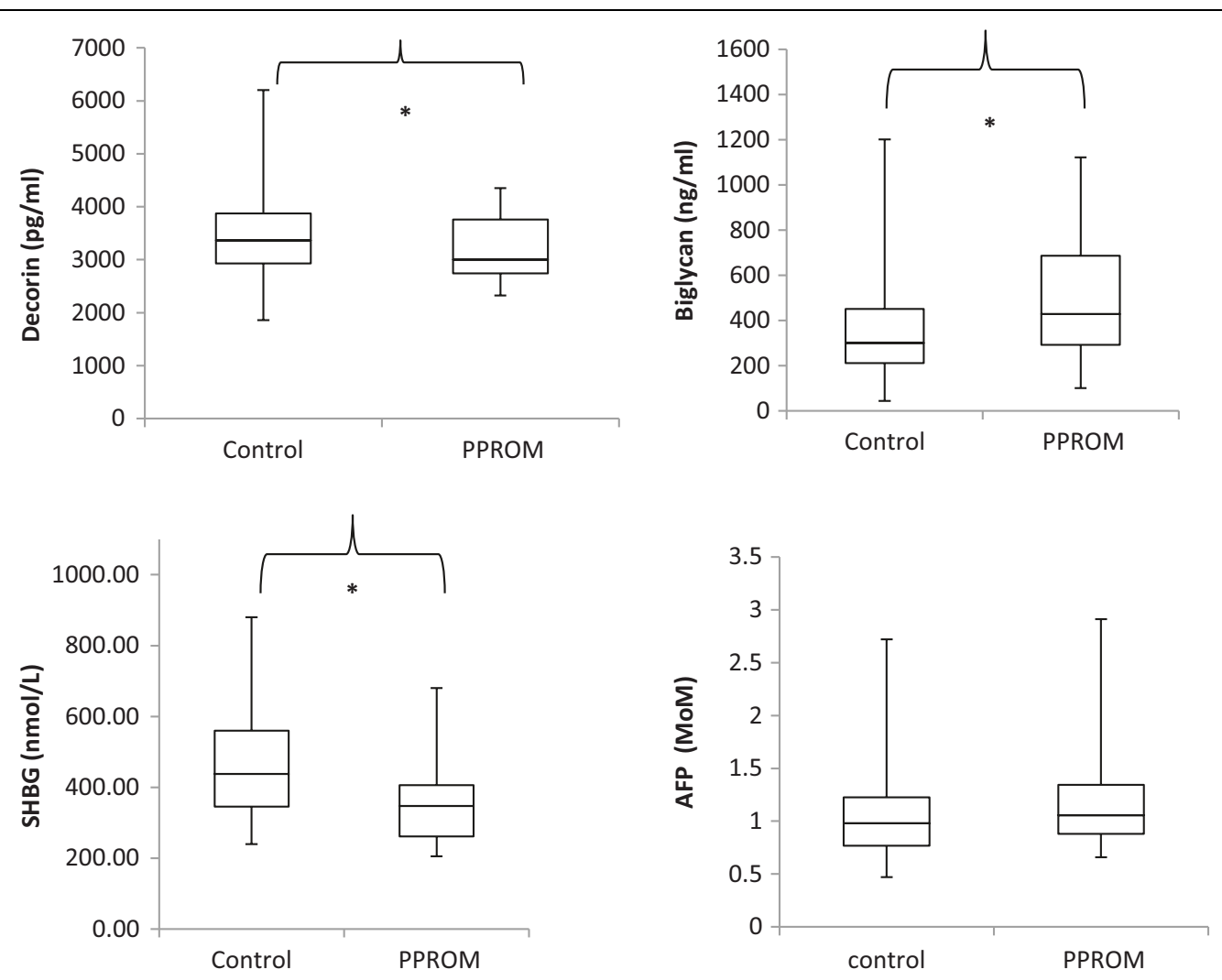

Figure 2. Serum biomarker levels in cases with preterm premature rupture of fetal membrane (PPROM) and controls. The median values for the proteoglycans were inversely correlated with biglycan significantly higher in the PPROM cases than the controls $(P<0.009)$, while decorin serum values were significantly lower in PPROM cases than in the controls $(P<0.009)$. The median serum concentrations of sex hormonebinding globulin (SHBG) presented significantly higher in controls versus cases with PPROM $(P<.006)$. Alpha-fetoprotein $(A F P)$ MoM serum concentrations were not significantly different in cases with PPROM compared to controls $(P=.153)$.

\section{Covariate Study}

Median serum levels of both biglycan and decorin were lower in pregnant $(\mathrm{n}=90)$ than in nonpregnant $(\mathrm{n}=15)$ women $(P<$ .002 ; Figure 1A). Levels of decorin were slightly lower in African American $(\mathrm{n}=15)$ than in non-African American women $(\mathrm{n}=15 ; P=.047)$, while biglycan levels did not differ by ethnicity $(P=.116$; Figure 1B). Based on the preanalytical and covariate results, the biomarker study samples were additionally matched for ethnicity and length of time in freezer storage.

\section{Biomarker Study}

The median maternal age in years was 28.55 (range: 18 - 40) for cases with PPROM and 28.77 (range: 17-42) for controls. Cases and controls were matched for gestational age with the median gestational age at the time of the blood serum sampling being 16.5 weeks for cases with PPROM and 16.4 weeks for controls. Mean latency between blood serum sampling and PPROM diagnosis was 40 days \pm 4.7 days.

Median second-trimester serum values of the biomarkers are presented in Table 1. A Box and Whiskers plot of the 4 biomarkers studied is presented in Figure 2. The median values for
Table 2. Analysis of Individual Biomarkers as Predictors of PPROM.

\begin{tabular}{lcc}
\hline Variable & AUC & $95 \% \mathrm{Cl}$ \\
\hline Biglycan & 0.659 & $0.513-0.806$ \\
Decorin & 0.563 & $0.412-0.714$ \\
SHBG & 0.726 & $0.590-0.862$ \\
Biglycan + Decorin + & 0.774 & $0.628-0.912$ \\
SHBG & &
\end{tabular}

Abbreviations: AUC, area under the curve; $\mathrm{Cl}$, confidence interval; PPROM, preterm premature rupture of fetal membrane; SHBG, sex hormone-binding globulin.

the proteoglycans were inversely correlated with biglycan significantly higher in the cases with PPROM than the controls $(P<.009)$, while decorin serum values were significantly lower in cases with PPROM than in the controls $(P<.009)$. The median serum concentrations of SHBG presented significantly higher in controls versus cases with PPROM $(P<.006)$. AFP MoM serum concentrations were not significantly different in cases with PPROM compared to controls $(P=.153)$. AFP is part of the routine screening panel conducted in second trimester to determine the risk of a number of disorders, including neural tube defects and down syndrome. Here, AFP serum 


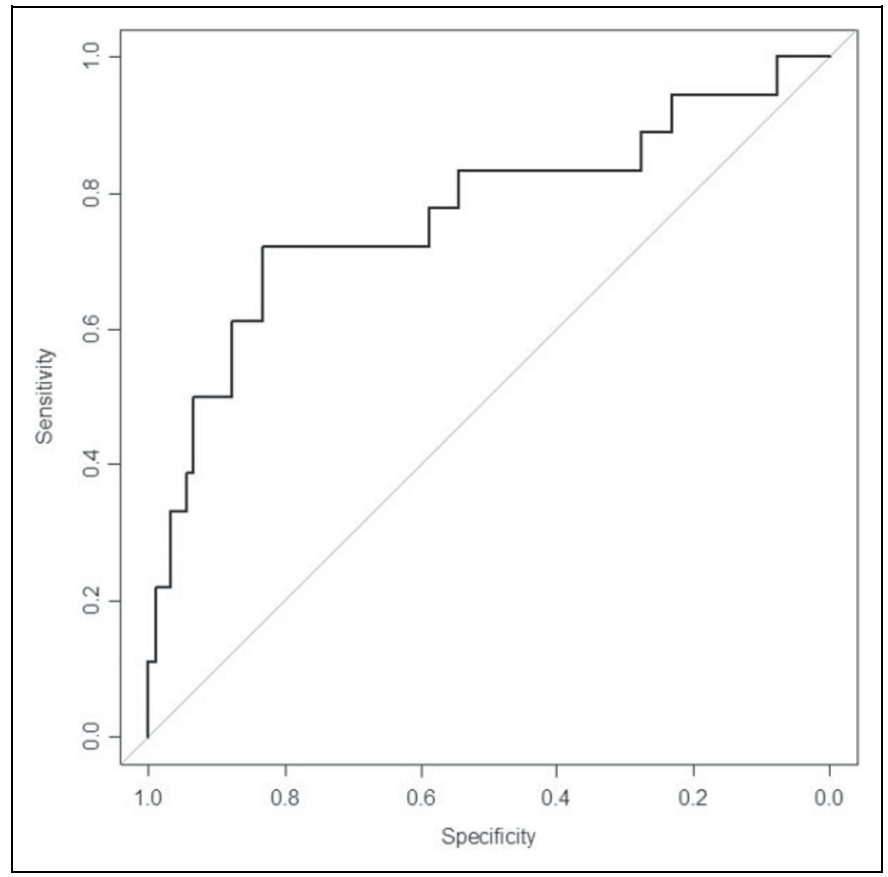

Figure 3. Receiver-operator characteristic (ROC) curve analysis of the predictive score for biglycan, decorin, and sex hormone-binding globulin (SHBG) as preterm premature rupture of fetal membrane (PPROM) serum biomarkers. The plot graphs sensitivity (truepositive rate) versus specificity (false-positive rate). The optimal threshold $(0.238)$, sensitivity $(0.72)$, and specificity $(0.84)$ were the same using both Youden index and top left criteria.

concentrations served to further validate that cases and controls were not complicated by fetal disorders that could have confounded pregnancy outcomes. Because AFP levels were not significantly different in cases versus controls, its inclusion in the predictive score was not statistically justified.

The 3 biomarkers, biglycan, decorin, and SHBG, were analyzed first separately and then in combination to determine their ability to predict PPROM. Individually, both SHBG and biglycan have AUC between 0.66 and 0.72 , while decorin (AUC = 0.56 ) alone is not as strong of a marker for predicting PPROM. The predictive score from the combined 3 variable model proves to be a very good test with AUC of 0.774 (Table 2).

The formula generated for the predicted score is $=1.52811$ $+0.00282 \times$ biglycan $-0.00051 \times$ decorin $-0.00647 \times$ SHBG. The ROC curve of the predicted score is seen in Figure 3. The optimal threshold (0.238), sensitivity (0.722), and specificity (0.844) were the same using both Youden index and top left criteria.

\section{Discussion}

In this study, elevated biglycan serum concentrations in conjunction with decreased decorin and SHBG serum concentrations in early pregnancy were observed for asymptomatic patients who later developed PPROM. These blood serum concentrations show an ability to predict the early development of PPROM in this study population.
The elevated biglycan serum concentrations we identified may represent early tissue damage in the fetal membrane of asymptomatic patients who later experience PPROM. Recent findings suggest that matrix-unbound biglycan acts as a danger signal, providing a link between the innate and the adaptive immune systems. ${ }^{28-30}$ Following tissue stress and injury, biglycan is proteolytically released from the ECM and acts as a damage-associated molecular pattern by activating Toll-like receptors, thus potentially modulating the immune system during sterile and pathogen-mediated inflammation events such as PPROM. ${ }^{31,32}$

Few studies are available on circulatory levels of decorin in human serum. Decreased plasma decorin levels have been reported in certain carcinomas as well as ischemic stroke. ${ }^{33-}$ ${ }^{35}$ Increased serum decorin levels have been reported in pregnancies with fetal growth restriction. ${ }^{36}$ Recently, a prospective study by Atalay et al investigated decorin levels in women 24 to 32 weeks gestation who presented to the hospital in labor. ${ }^{37}$ In this study, decreased serum levels of decorin were determined in patients with PTL compared to healthy pregnancies. The authors concluded that serum decorin levels had no effect in predicting preterm births before 34 weeks gestation in women with PTL. Our results showed a decrease in decorin levels during the second trimester (weeks 13-28) of asymptomatic patients who later presented with PPROM. Therefore, our results indicate serum decorin levels have an effect in predicting PPROM.

Elevated circulatory levels of biglycan in conjunction with decreased decorin have been reported in urothelial carcinoma of the bladder, but further analysis of the ROCs for the SLRPs revealed serum lumican provided the optimum sensitivity and specificity as a potential biomarker for this disease. ${ }^{33}$

Our findings that second-trimester SHBG serum concentrations were lower in patients who later developed PPROM has not been previously reported. Decreased SHBG levels in the second trimester have been reported as a predictor of preeclampsia development, but the area under the ROC curve was low, resulting in a poor predictor model. ${ }^{38}$ SHBG levels have also been shown to be significantly reduced in women whose pregnancy results in miscarriage. ${ }^{39}$ More recently, Saade et al have developed a maternal serum predictor for spontaneous preterm delivery resulting from the ratio of downregulated SHBG to insulin-like growth factor-binding protein 4 with an elevated body mass index (BMI). ${ }^{22}$ It is not surprising that SHBG is downregulated in both SPTB and PPROM, since PPROM is the causal factor for a third of SPTBs.

It is well established that inflammation is a process associated with preterm birth and PPROM. It has been reported that upregulation of proinflammatory cytokines Interleukin-6, Interleukin- 8 , and tumor necrosis factor- $\alpha$ play a significant role in PPROM. ${ }^{16,26}$ Furthermore, maternal serum cytokine levels have been reported to be elevated in women who later deliver preterm. ${ }^{40}$

A study by Kansu-Celik et al evaluated the role of maternal serum advanced glycation end products (AGEs) in early gestation for prediction of PTB. They determined that AGEs are 
significantly elevated in preterm births due to PTL and/or PPROM. ${ }^{41}$ Increased levels of AGE have previously been associated with the expression of inflammatory cytokines in preeclampsia as well. ${ }^{42}$ Thus, while AGEs may play a role in poor pregnancy outcomes, they do not seem to be discriminate to PTB due to PPROM.

A caveat to this study is that the retrospective study design does not allow for detailed analysis of the clinical overlap between PPROM and preterm birth. Additionally, several studies have associated maternal BMI with risk of preterm birth. ${ }^{43-46}$ Thus, future prospective studies will be necessary to verify our model as a clinically viable specific biomarker for PPROM in which the clinical history of the timing between onset of contractions and PPROM are prospectively collected and in which the association of BMI to PPROM can be explored.

In summary, a second-trimester prenatal serum panel consisting of the SLRPs biglycan and decorin with SHBG is promisingly the first serum screening-based biochemical model to predict PPROM in asymptomatic women. This model has the potential to open the door to a new approach to identifying women who are at increased risk of PPROM and targeting future preventive interventions in a personalized manner.

\section{Declaration of Conflicting Interests}

The author(s) declared no potential conflicts of interest with respect to the research, authorship, and/or publication of this article.

\section{Funding}

The author(s) disclosed receipt of the following financial support for the research, authorship, and/or publication of this article: This work was supported by the National Institute of General Medical Sciences COBRE for Reproductive Health [P20GM121298].

\section{References}

1. Goldenberg RL, Culhane JF, Iams JD, Romero R. Epidemiology and causes of preterm birth. Lancet. 2008;371(9606):75-84.

2. World Health Organization. Born too Soon; The Global Action Report on Preterm Birth. Geneva, Switzerland: World Health Organization: March of DImes, PMNCH, Save the Children; 2012.

3. Murphy SL, Xu J, Kochanel KD. Deaths: Preliminary Data for 2010. U.S. Melbourne, Australia: Dept Health and Human Services; 2012.

4. Mercer B. Preterm premature rupture of the membranes. Obstet Gynecol. 2003;101(1):178-193.

5. Mingione MJ, Pressman EK, Woods JR. Prevention of PPROM: current and future strategies. J Matern Fetal Neonatal Med. 2006; 19(12):783-789.

6. Tchirikov MS-L, Maher J, Buchmann J, Naberezhnev Y, Winarno AS, Seliger G. Mid-trimester preterm premature rupture of membranes (PPROM); etiology, diagnosis, classification, international recommendtions of treatment options, and outcome. $J$ Perinat Med. 2018;46(5):465-488.

7. Mercer BM, Goldenberg RL, Meis PJ, et al. The preterm prediction study: prediction of preterm premature rupture of membranes through clinical findings and ancillary testing. Am J Obstet Gynecol. 2000;183(3):738-744.

8. Romero RMJ, Chaemsaithong P, Chaiworapongsa T, et al. Sterile and microgial-associated intra-amnionic inflammation in preterm premature rupture of membrane. J Matern Fetal Neonatal Med. 2015;28(12):1394-1409.

9. Barabas AP. Ehlers-Danlos syndrome: associated with prematurity and premature rupture of foetal membranes; possible increase in incidence. Br Med J. 1966;2(5515):682-684.

10. Yen JL, Lin SP, Chen MR, Niu DM. Clinical features of EhlersDanlos syndrome. J Formos Med Assoc. 2006;105(6):475-480.

11. Quentin E, Gladen A, Roden L, Kreses H. A genetic defect in the biosynthesis of dermatan sulfate proteoglycan: galactosyltransferase 1 deficiency in fibroblasts from a patient with a progeroid syndrome. Proc Natl Acad Sci U S A. 1990;87(4):1342-1346.

12. Ameye L, Young MF. Mice deficient in small leucine-rich proteoglycans: novel in vivo models for osteoporosis, oseoarthritis, Ehlers-Danlos syndrome, muscular dystrophy, and corneal diseases. Glycobiology. 2002;12(9):107R-116R.

13. Westermann D, Mersmann J, Melchior A, et al. Biglycan is required for adaptive remodeling after myocardial infarction. Circulation. 2008;117(10):1269-1276.

14. Zhang G, Chen S, Goldoni S, et al. Genetic evidence for the coordinated regulation of collagen fibrillogenesis in the cornea by decorin and biglycan. J Biol Chem. 2009;284(13):8888-8897.

15. Schaefer L, Babelova A, Kiss E, et al. The matrix component biglycan is proinflammatory and signals through Toll-like receptors 4 and 2 in macrophages. J Clin Invest. 2005;115(8): 2223-2233.

16. Parry S, Strauss JF. Premature rupture of the fetal membranes. N Engl J Med. 1998;338(10):663-670.

17. de Miranda de Araujo LB, Horgan CE, Aron A, Iozzo RV, Lechner BE. Compensatory fetal membrane mechanisms between biglycan and decorin in inflammation. Mol Reprod Dev. 2015; 82(5):387-396.

18. Meinert M, Malmström A, Petersen AC, Eriksen GV, Uldbjerg N. Chorioamnionitis in preterm delivery is associated with degradation of decorin and biglycan and depletion of hyaluronan in fetal membranes. Placenta. 2014;35(8):546-551.

19. Horgan CE, Roumimper H, Tucker R, Lechner BE. Altered decorin and Smad expression in human fetal membranes in PPROM. Biol Reprod. 2014;91(5):105,101-107.

20. Calmus ML, Macksoud EE, Tucker R, Iozzo RV, Lechner BE. A mouse model of spontaneous preterm birth based on the genetic ablation of biglycan and decorin. Reproduction. 2011;142(1): 183-194.

21. Wu Z, Horgan CE, Carr O, Owens RT, Iozzo RV, Lechner BE. Biglycan and decorin differentially regulate signaling in the fetal membranes. Matrix Biol. 2014;35:266-275.

22. Saade GR, Bogess KA, Sullivan SA, et al. Development and validation of a spontaneous preterm delivery predictor in asymptomatic women. Am J Obstet Gynecol. 2016;21(4):633e631$633 \mathrm{e} 624$.

23. Anderson DC. Sex-hormone-binding globulin. Clin Endocrinol. 1974;3(1):69-93. 
24. Savitz D, Dole N, Herring A, et al. Should spontaneous and medically indicated preterm births be separated for studying aetiology? Paediatr Perinat Epidemiol. 2005;19(2):97-105.

25. Dutta EH, Behnia F, Boldogh I, et al. Oxidative stress damageassociated molecular signaling pathways differentiate spontaneous preterm birth and preterm premature rupture of the membranes. Mol Hum Reprod. 2016;22(2):143-157.

26. Menon R, Richardson LS. Preterm prelabor rupture of the membranes: a disease of the fetal membranes. Semin Perinat. 2017; 41(7):409-419.

27. DeLong E, DeLong DM, Clarke-Pearson DL. Comparing the areas under two or more correlated receiver operating characteristic curves: a nonparametric approach. Biometrics. 1988;44(3): 837-845.

28. Frey H, Schroeder N, Manon-Jensen T, Iozzo RV, Schaefer L. Biological interplay between proteoglycans and their innate immune receptors in inflammation. FEBS J. 2013;280(10): 2165-2179.

29. Moreth K, Iozzo RV, Schaefer L. Small leucine-rich proteoglycans orchestrate receptor crosstalk during inflammation. Cell Cycle. 2012;11(11):2084-2091.

30. Zeng-Brouwers J, Beckmann J, Nastase M-V, Iozzo RV, Schaefer L. De novo expression of circulating biglycan evokes an innate inflammatory tissue response via MyD88/TRIF pathway. Matrix Biol. 2014;35:132-142.

31. Nastase MV, Young MF, Schaefer L. Biglycan: a multivalent proteoglycan providing structure and signals. $J$ Histochem Cytochem. 2012;60(12):963-975.

32. Iozzo RV, Schaefer L. Proteoglycans in health and disease: novel regulatory signaling mechanisms evoked by the small leucinerich proteoglycans. FEBS J. 2010;277(19):3864-3875.

33. Appunni S, Anand V, Khandelwal M, Seth A, Mathur S, Sharma A. Altered expression of small leucine-rich proteoglycans (decorin, biglycan and lumican): plausible diagnostic marker in urothelial carcinoma of bladder. Tumour Biol. 2017;39(5):1-10.

34. Xu YZ, Yang ZG, Zhang YH, Zhang YW, Hong B, Liu JM. Dynamic reduction of plasma decorin following ischemic stroke: a pilot study. Neurochem Res. 2012;37(9):1843-1848.

35. Wu I-C, Wu D-C, Huang C-C, et al. Plasma decorin predicts the presence of esophageal squamous cell carcinoma. Int $J$ Cancer. 2010;127(21):2138-2146.
36. Caglar M, Yavuzcan A, Goksu M, et al. Decorin: a possible marker for fetal growth restriction. Gynecol Endocrinol. 2014; 30(2):141-144.

37. Atalay MA, Ozmen T, Demir BC, Kasapoglu I, Ozkaya G. Serum decorin measurement in prediction of the risk for preterm birth. Taiwan J Obstet Gynecol. 2018;57(1):23-27.

38. Yu CK, Papageorghiou AT, Binda R, Spencer K, Nicolaides KH. Second-trimester sex hormone-binding globulin and subsequent development of pre-eclampsia. J Matern Fetal Neonatal Med. 2004;16(3):158-162.

39. Spencer K, Yu CK, Rembouskos G, Bindra R, Nicolaides KH. First trimester sex hormone-binding globulin and subsequent development of preeclampsia or other adverse pregnancy outcomes. Hypertens Pregnancy. 2005;24(3):303-311.

40. Herrera-Munoz A, Fernandez-Alonso AM, Fischer-Suarez N, Chedraui P, Perez-Lopez FR. Maternal serum cytokine levels in pregnancies complicated with threatened preterm labor. Gynecol Endocrinol. 2017;33(5):408-412.

41. Kansu-Celik H, Tasci Y, Karakaya BK, Cinar M, Candar T, Caglar GS. Maternal serum advanced glycation end products level as an early marker for predicting preterm labor/PPROM: a prospective study. J Matern Fetal Neonatal Med. 2018;22:1-5.

42. Chen W, Zhang Y, Yue C, et al. Accumulation of advanced glycation end products involved in inflammation and contributing to severe preeclampsia in maternal blood, umbilical blood and placental tissues. Gynecol Obstet Invest. 2017;82(4):388-397.

43. Hong X, Hao K, Ji H, et al. Genome-wide approach identifies a novel gene-maternal pre-pregnancy BMI interaction on preterm birth. Nat Commun. 2017:1-9.

44. Torloni MR, Betran AP, Daher S, et al. Maternal BMI and preterm birth: a systematic review of the literature with met-analysis. J Matern Fetal Neonatal Med. 2009;22(11):957-970.

45. Shaw GM, Wise PH, Mayo J, Carmichael SL, Ley C, Lyell DJ, et al. Maternal prepregnancy body mass index and risk of spontaneous preterm birth. Paediatr Perinat Epidemiol. 2014; 28(4):302-311.

46. Andraweera PH, Dekker GA, Thompson SD, et al. The interaction between the maternal BMI and angiogenic gene polymorphisms associates with the risk of spontaneous preterm birth. Mol Hum Reprod. 2012;18(9):459-465. 